\title{
The Thickness of the Sternocleidomastoid Muscle as a Prognostic Factor for Congenital Muscular Torticollis
}

\author{
Jae Deok Han, M.D., Seung Hwan Kim, M.D. ', Seung Jae Lee, M.D., \\ Myong Chul Park, M.D. ${ }^{2}$, Shin-Young Yim, M.D. \\ Department of Physical Medicine and Rehabilitation, Ajou University School of Medicine, Suwon 442-749, \\ ${ }^{1}$ Hwaseong City Health Center, Hwaseong 445-920, ${ }^{2}$ Department of Plastic and Reconstructive Surgery, \\ Ajou University School of Medicine, Suwon 442-749, Korea
}

Objective To examine whether the thickness of the sternocleidomastoid muscle (SCM) could be used as a prognostic factor for congenital muscular torticollis (CMT).

Method This was a retrospective study conducted in a pediatric rehabilitation service at a tertiary medical center. Fifty-two children who met the following inclusion criteria were included: 1) children who were 3 month-old or younger, 2) children diagnosed with CMT, 3) passive rotation of the face toward the shoulder of the tilted side $\leq 60^{\circ}$, 4) children who had been managed according to the clinical pathway for CMT, 5) children who had been followed up for 6 months or more after the end of treatment. The duration and total number of stretching exercise sessions were reviewed with reference to the thickness of the SCM.

Results Among the 52 children with CMT, 46 children were successfully managed with only stretching exercise of the SCM for 1-6 weeks (group 1: 88.5\%) and 6 children were managed with botulinum toxin A injection, surgical release or both in addition to stretching exercise (group 2: 11.5\%). The difference in the SCM thickness between the affected and normal sides was significantly greater in group 2 than that in group $1(\mathrm{p}=0.026)$. A strong correlation was found between the total duration of stretching exercise and the difference in the SCM thickness in group 1 (Pearson' $\gamma=0.429$; $p=0.003$ ).

Conclusion Children with a thicker SCM seem to require a longer duration of stretching exercise and other therapeutic interventions in addition to stretching exercise for CMT. Therefore, the thickness of the SCM may be one prognostic factor for CMT treatment.

Key Words Torticollis, Sternocleidomastoid, Ultrasonography, Prognosis

Received June 22, 2010; Accepted November 18, 2010

Corresponding author: Shin-Young Yim

Department of Physical Medicine and Rehabilitation, Ajou University School of Medicine, San $5^{\text {th }}$, Wonchon-dong, Yeongtong-gu, Suwon 442749 , Korea

Tel: +82-31-219-5284, Fax: +82-31-219-5209, E-mail: syyim@ajou.ac.kr Copyright $\odot 2011$ by Korean Academy of Rehabilitation Medicine

\section{INTRODUCTION}

Congenital muscular torticollis (CMT) is the most common musculoskeletal disorder in infancy. ${ }^{1}$ According to the diagnostic method and target population, the reported incidence of CMT varies from $0.0084-3.92 \%$ of 
all newborns. ${ }^{2-8} \mathrm{CMT}$ occurs during or before the delivery, due to a shortened unilateral sternocleidomastoid muscle (SCM), and typically the head is tilted to the shortened muscle and at the same time the chin faces to the opposite direction. ${ }^{1,4,9-12}$

The first line treatment for CMT in neonates is physical therapy, including stretching exercise. Through stretching, $>90 \%$ of the neonates reach full recovery without complications such as craniofacial deformation, but some groups do not reach full recovery even with an early sufficient period of stretching exercise. ${ }^{1,13-15}$ Surgical treatment has traditionally been performed for these groups. The recent research on botulinum toxin type A injection has reported different results and effects. ${ }^{16-18}$

Cheng et al. ${ }^{13}$ reported that the average age of 510 children when they were diagnosed with CMT was 24 days (range: 10-299 days), and the age of symptom onset was one of the predictors of a response to a stretching exercise program. Yet few studies have investigated the prognostic factors of successful early stretching exercise for the CMT children diagnosed before the age of 3 months. ${ }^{19}$

Ultrasonographic evaluation of CMT is the most widely employed method to obtain the primary diagnostic image, and making the diagnosis of CMT is possible by scanning and comparing the difference in the thickness of the SCMs of infants.

Our clinical experience led us to hypothesize about the prognosis of CMT, that is, if the thickness of the SCM, as evaluated by ultrasonography, was a prognostic factor for successful stretching exercise, then this could help clinicians make the diagnosis, as well as guide the course of treatment. If the shortened SCM is thicker than the criteria at the time of diagnosis, then we could predict that stretching exercise alone would not cause complete recovery. Although patients do recover with stretching exercise only, perhaps we can predict that the thicker the SCM is, the longer the required period of stretching exercise. This can be very useful predictive information for both the clinician and patients.

The objective of this study was to investigate whether the thickness of the SCM, as examined by ultrasound, is a prognostic factor for the management of early-diagnosed CMT (before 3 months of age).

\section{MATERIALS AND METHODS}

\section{Subjects}

The subjects in this study were infants who were treated for abnormal posturing of the head and neck in the Center for Torticollis at the Department of Physical Medicine and Rehabilitation from 2003 to 2006. Among the 246 children who were treated, the inclusion criteria were 1) children with CMT, 2) age $>3$ months at presentation, 3) neck passive rotation was at least 60 degree to the mass side in the relaxed position, 4) subjects were treated according to the clinical pathway (Fig. 1), and had been followed up for $>6$ months at the end of treatment. The exclusion criteria were 1) children with CMT and who had previous manual stretching exercise done before they came to our clinic, 2) children with neurodevelopmental conditions that could cause a delay of head control, and 3) children with any inflammatory and/or infectious diseases of the neck.

The diagnosis of CMT was made when the following criteria were simultaneously met: 1 ) the head was tilted toward the shoulder of one side and the chin was turned toward the shoulder of the opposite side, and 2) the SCM muscle of the involved side was $>2 \mathrm{~mm}$ thicker than that of the non-tilted side as shown on ultrasonography.

\section{Treatment of CMT}

The children were treated according to our clinical pathway for CMT (Fig. 1). As first-line therapy, all the children diagnosed before 3 months were treated with manual stretching exercise for the shortened SCM by therapists as an outpatient 3-5 days per week. Each session of physical therapy consisted of passive rotation, lateral flexion of the neck to stretch the shortened SCM, therapeutic ultrasound for the shortened SCM, and massage for the mass for 20 to 30 minutes a day by physical therapists with $>10$ years experience. In addition to manual stretching exercise by a therapist, a caregiver was taught stretching exercise, and this person was encouraged to give one or two sessions of manual stretching exercise at home per day.

When there was no definite resistance during the rotation of the face, and it could be rotated to 90 degrees to the mass side, and simultaneously there was no resistance during lateral flexion of the face to the nonmass side as compared with that of the other side, the 


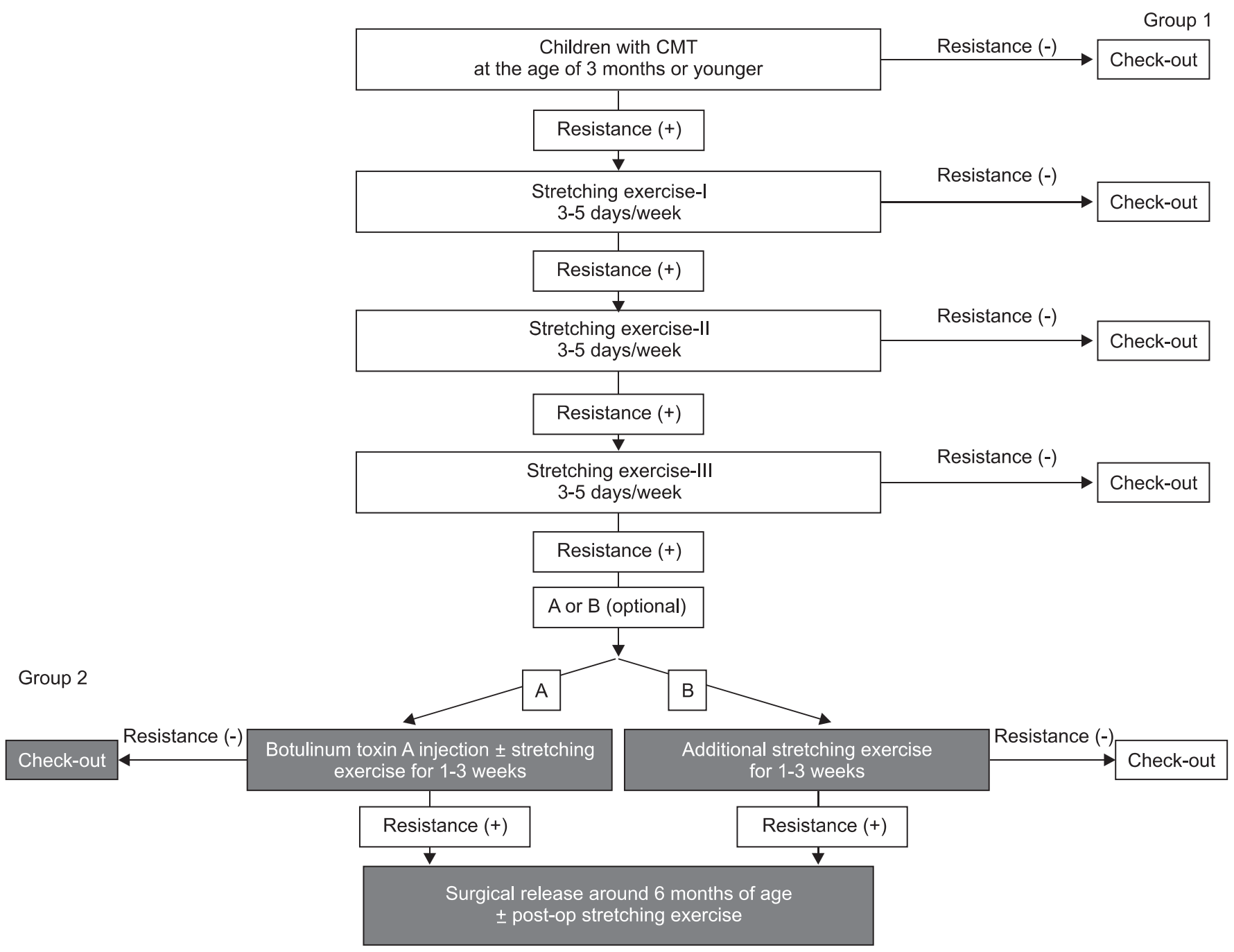

Fig. 1. Clinical pathway for treatment of children with congenital muscular torticollis.

management was considered successful and it was terminated regardless of the presence of the neck mass of CMT. If the head tilt was left in the coronal plane, but rotation and flexion of the neck on passive motion had no limit, then we judged that the tilted head was not due to a shortened SCM and we ended treatment.

Despite of stretching for up to 4-6 weeks, if there were limitations of neck flexion and rotation, and a significant reduction in the SCM muscle mass thickness was not observed, we thought the head asymmetry was likely to progress, and the patient was treated as follows: 1) Type A botulinum toxin was injected into the shortened SCM, 2) surgical release for the shortened SCM, and 3) Type A botulinum toxin injection and surgical release. Botulinum toxin type A $\left(\right.$ BOTOX $^{\circledR}$, Allergan Inc., California, USA) mixed with $0.9 \%$ saline and diluted to a total of $2 \mathrm{cc}(2-4$ units $/ \mathrm{kg})$ was injected into the muscle mass using 27-gauge and $30 \mathrm{~mm}$ needles and a $1 \mathrm{cc}$ syringe. If surgery was necessary, from 6 months of age, the shortened SCM was released by the third author of this study. A transverse $1.5 \mathrm{~cm}$ skin incision was made 1 $\mathrm{cm}$ above and between the sternal and clavicular ends of the SCM. The contracted fibrous tissue and contracted myofascia were released and the sternal end, clavicular end, or both ends were released on a case by case basis using bipolar electrocautery.

Therefore, by the clinical pathway, the children whose treatment was terminated after only stretching exercise (group 1) were treated during a different period of time within 6 weeks of terminating the stretching exercise, depending on the child's reaction. Group 2 is shown in (Fig. 1). After completing the stretching exercise-III 
without recovery and choosing the patients who received an injection of botulinum toxin type A, stretching exercise by the therapist was made optional after the injection. If the children did not recover, the shortened SCM was released after 6 months of age. Clinical pathway B: If the patient did not recover by stretching exercise III, and there was sustained resistance after 3 additional weeks of stretching exercise by the therapist, the shortened SCM was released after 6 months of age. Therefore, the total duration and number of sessions of stretching by the therapist was quantified in group 1 , but not in group 2 .

\section{Measurement of the SCM thickness}

On the ultrasonography performed at presentation, the thickness of the SCM was measured at the thickest point of the SCM on both sides on the longitudinal view of ultrasonography. The differences of thickness of the SCMs were compared between the two groups: the patients who successfully ended treatment only with stretching exercise within 6 weeks (group 1), and the patients for whom stretching exercise alone did not end treatment, so botulinum toxin type A injection and/or surgical release was done in addition to stretching exercise (group 2). The difference of thickness was calculated from the data of the measured right and left SCMs, and the ratio of the thickness of the SCMs was defined as the thickness of the shortened SCM/contralateral SCM thickness. In addition, the duration of stretching exercise and the total number of sessions of stretching exercise were reviewed. Then, the relationships between the mass thickness, the duration of stretching, and the total number of treatment sessions were analyzed.

\section{Statistical analysis}

SPSS 11.5 for Windows was used for all the statistical analyses in this study. Independent t-tests were used to compare the thickness of the SCMs between group 1 and group 2. Correlation analysis with Pearson's $\gamma$ correlation coefficient was used for group 1 to evaluate the relationship between the difference of thickness between the SCMs and the duration of stretching exercise. A predictive linear regression model was constructed through linear regression analysis for group 1 to determine the treatment outcome. The level for significance for all the statistical analyses was set at $\mathrm{p}=0.05$.

\section{RESULTS}

Comparison between group 1 and group 2 (Table 1, 2)

Fifty-two children ( 32 boys and 20 girls, age at presentation: $1.14 \pm 0.66$ month-old, range: 13 day-old

Table 1. Comparison of Group 1 and Group 2

\begin{tabular}{lccc}
\hline \multirow{2}{*}{ Variables } & \multicolumn{2}{c}{ Mean \pm SD (range) } & p-value \\
\cline { 2 - 3 } & Group 1 (n=46) & Group 2 (n=6) & 0.319 \\
\hline Age at presentation (days) & $32.4 \pm 17.1(13-90)$ & $47.7 \pm 33.3(16-90)$ & 0.026 \\
Side-to-side SCM muscle thickness difference (mm) & $6.6 \pm 2.2(2.6-10.3)$ & $8.8 \pm 2.0(6.8-12.5)$ & 0.730 \\
The ratio of the SCMs & $2.33 \pm 0.53(1.48-3.70)$ & $2.77 \pm 0.72(2.23-4.21)$ & 0.730 \\
\hline
\end{tabular}

Side-to-side SCM muscle thickness ratio: the thickness of the shortened SCM/the thickness of the contralateral SCM

Table 2. Characteristics of the Subjects of Group $2(\mathrm{n}=6)$

\begin{tabular}{|ccc}
\hline Subject & $\begin{array}{c}\text { Gender/Age (months) } \\
\text { at the first visit }\end{array}$ & \multicolumn{1}{c}{ Therapeutic methods } \\
\hline Subject 1 & $\mathrm{~F} / 0.6$ & Stretching exercises + botulinum toxin A injection \\
\hline Subject 2 & $\mathrm{M} / 1$ & \\
\hline Subject 3 & $\mathrm{~F} / 1$ & Stretching exercises + surgical release \\
\hline Subject 4 & $\mathrm{~F} / 3$ & Stretching exercises + botulinum toxin A injection \\
\hline Subject 5 & $\mathrm{M} / 1$ & + surgical release \\
\hline Subject 6 & $\mathrm{M} / 3$ & \\
\hline
\end{tabular}


Table 3. Intercorrelations for the Stretching Exercise Parameters and the Patient Variables in Group 1 ( $\mathrm{n}=46$ )

\begin{tabular}{lccc}
\multicolumn{1}{c}{$\begin{array}{c}\text { Stretching exercise } \\
\text { parameters }\end{array}$} & SCM difference & SCM ratio & Age at presentation \\
\hline Total duration & $0.429^{*}$ & $0.427^{*}$ & $-0.108^{*}$ \\
Total number & $0.404^{\dagger}$ & $0.403^{\dagger}$ & $-0.085^{\dagger}$ \\
\hline
\end{tabular}

SCM difference: Side-to-side SCM muscle thickness difference, SCM ratio: Side-to-side SCM muscle thickness ratio ${ }^{*} \mathrm{p}=.003$ for Pearson's $\gamma$ correlation coefficient, ${ }^{\dagger} \mathrm{p}=.005,{ }^{\dagger} \mathrm{p}>0.05$

-3 month-old) were included in this study. Among the 52 children with CMT, 46 children (group I: 88.5\%) were successfully managed with only stretching exercise of the SCM muscle for $<6$ weeks, and they did not show recurrence through 6 months of follow-up.

Among the 52, 6 children (group 2: 11.5\%) were managed with botulinum toxin A injection and/or surgical release in addition to stretching exercise, and the normal neck range of motion was recovered (Table 2). The difference of thickness between the SCMs ( $\mathrm{p}=0.026$, $6.6 \pm 2.2 \mathrm{~mm}$ in group 1 , and $8.8 \pm 2.0 \mathrm{~mm}$ in group 2) was significantly greater in group 2 than in group 1 (Table 1 ; $\mathrm{p}<0.05$ ). Among the 52 children, the normal side of SCM thickness was $5.21 \pm 0.80 \mathrm{~mm}$, so if the thickness of the mass side SCM was larger $(>8.8 \mathrm{~mm})$ than the normal side, or the shortened SCM thickness was $>14.01 \mathrm{~mm}$ $(5.21+8.8 \mathrm{~mm})$, then treatment other than stretching exercise was required. However, there were no significant differences between group 1 and group 2 for the age of presentation and the ratio of the SCM thickness (Table 1, $\mathrm{p}>0.05)$.

Correlation analysis and linear regression analysis of the total duration of stretching exercise and the SCM thickness difference in group 1 (Table 3, Fig. 2)

Group A included 46 patients who recovered with only stretching exercise and they were treated for a total of $19.76 \pm 15.33$ days, and with $11.54 \pm 8.59$ sessions of stretching exercise. The correlation analysis performed for these factors showed that the total period of stretching exercises (Pearson $\gamma$ correlation coefficient: 0.429, $\mathrm{p}=0.003$ ) and the total number of stretching exercises (Pearson $\gamma$ correlation coefficient: $0.404, \mathrm{p}=0.005$ ) showed significant correlation with the differences in the thickness of the SCM. Also, the SCM thickness ratio showed significant correlation with the total duration of the stretching exercises (Pearson $\gamma$ correlation coefficient:

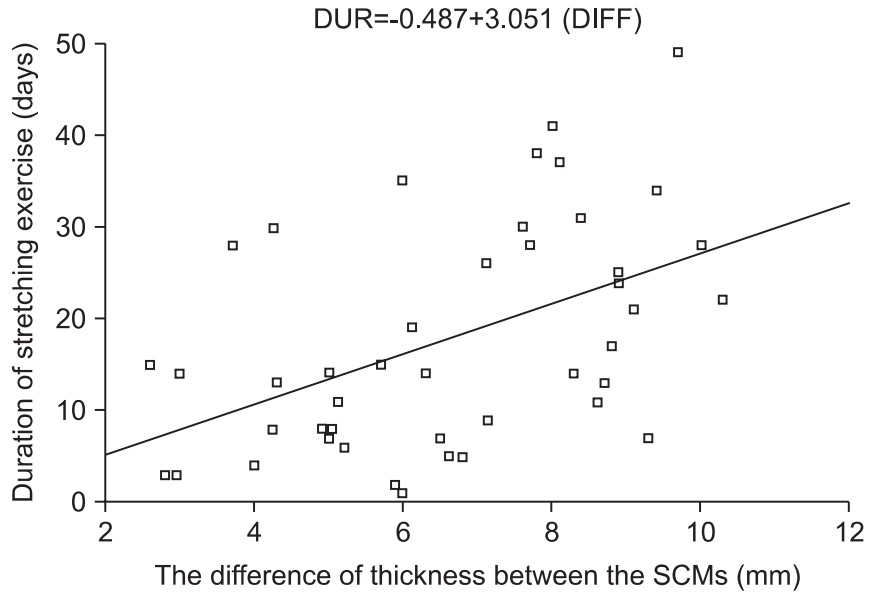

Fig. 2. Regression analysis of the duration of stretching exercise and the side-to-side sternocleidomastoid (SCM) muscle thickness difference (DIFF) in group 1. Duration of stretching exercises for CMT (DUR in days) $=-0.487+3.051$ (DIFF in $\mathrm{mm}$ ) with an adjusted $\mathrm{R}^{2}$ of 0.166 ( $\left.\mathrm{p}=0.003\right)$.

$0.427, \mathrm{p}=0.003)$, and the total number of relaxation exercise sessions (Pearson $\gamma$ correlation coefficient: $0.403, \mathrm{p}=0.005$ ). Therefore, in group 1 (patients who recovered with only stretching exercise), results showed that the larger the size of the mass, the longer the period of treatment and the greater the number of treatment sessions (Table 3). However, the age at presentation had no significant correlation with the duration of stretching exercise and the total number of treatment sessions (Table $3, p>0.05$ ). Correlation analysis showed more significant correlation between the duration of stretching exercises and the difference of thickness between the SCMs (0.429 of Pearson $\gamma$ correlation coefficient: $0.429, \mathrm{p}=0.003$, Table 3 ). The linear regression analysis (Fig. 2) of the duration of stretching exercise for CMT with the difference of thickness between the SCMs (DIFF) in group I showed the following equation with an adjusted $\mathrm{R}^{2}$ of 0.166 $(\mathrm{p}=0.003)$ : The duration of stretching exercises for $\mathrm{CMT}=$ $-0.487+3.051$ (DIFF). 


\section{DISCUSSION}

This study revealed that a thicker SCM on the tilted side seemed to require a longer duration of stretching exercise and other therapeutic interventions in addition to stretching exercise for children with CMT and who were diagnosed before 3 months of age.

Lim et al. ${ }^{19}$ recently reported that children with CMT required $>12$ months of stretching exercise and their ultrasonographic thickness ratio of the SCMs (shortened SCM thickness/normal SCM thickness) was 3.38 (range: 2.45 to 4.31 ). The children with CMT required $<12$ months of stretching exercise, their ultrasonographic thickness ratio was 2.04 (range: 1.14 to 2.94), and the results of this current study were in agreement with Lim et al.'s results. However, in that previous study, they did not comment about a necessity for other treatments, such as surgical release, besides stretching exercise. Ultrasonography is the most common modality used to diagnose CMT, and is also used for the follow-up to judge the effectiveness of stretching exercise. ${ }^{2,20-24}$

Cheng et al. ${ }^{20}$ reported that for children with CMT, when diagnosed by ultrasonography, the ratio of the upper $1 / 3$ and lower $1 / 3$ of the shortened SCM thickness over the normal side was correlated with the degree of cervical rotation restriction. Accordingly, those authors addressed the relation between the SCM thickness and the severity of symptoms at the time of diagnosis, but they did not mention the correlation with stretching exercise as a prognostic factor.

In this study, for the children with CMT with an age at the time of diagnosis of $<3$ months, the thickness of the SCM could be the prognostic factor for the management of CMT. It is well known that the majority of children with CMT are brought for management before the age of 3 months ${ }^{13}$ Further studies are warranted to compare the prognosis of children with CMT and whose age is $>3$ month-old, with that of children with CMT and whose age is $<3$ months.

Stretching exercise of the shortened SCM is known as the first-line management for CMT, yet chemodenervation by using botulinum toxin and surgical release can be used for the recalcitrant cases. ${ }^{16,17}$ To the best of our knowledge, there is no proposed therapeutic guideline for CMT using manual stretching exercise, botulinum toxin A injection, and surgery. Therefore, we had to develop our own clinical pathway (Fig. 1) for the children with CMT who were 3 month-old or younger. Our study with this clinical pathway showed that $88.5 \%$ of the children were successfully treated with only manual stretching exercises, with a mean duration of 19.86 days. The remaining children (11.5\%) needed other therapeutic options such as botulinum toxin A injection, and/or surgical release even after manual stretching exercise for up to 3-5 weeks.

Celayir $^{25}$ reported the treatment outcome of 45 children with CMT whose mean age was 38.6 days (range: 15120 days) old at initial assessment. All the children were successfully treated with an intensive home treatment protocol of passive stretching exercises, with a mean treatment duration of 3.2 months. These results involved a relatively longer term of stretching exercise to produce complete recovery, as compared to that of our study.

This current study revealed a relatively higher rate of botulinum toxin A injection \pm surgical release (11.5\%) than that of the reports by Celayir ${ }^{25}$ and Cheng et al., ${ }^{13}$ and this result could be related to the relatively short duration of manual stretching exercise (up to a little less than 6 weeks). There is some dispute regarding the proper timing for performing surgical release of the SCM in children with $\mathrm{CMT} .^{10,26-28}$

Cheng et al. recommended that surgical treatment should be considered when a patient underwent at least 6 months of controlled manual stretching and the patient still had residual head tilt, deficits of passive rotation and lateral bending of the neck $>15^{\circ}$, and a tight muscular band or tumor. ${ }^{22}$ However, as children get older, they get more strength in their body including the neck, and their waking hours during the day get longer. Once a child reaches the milestones of head control and rollingover, (-age of 3-4 months), implementation of manual stretching exercises is not always easy to accomplish because of the child's physical and emotional resistance.

Moreover, Yu et al. ${ }^{29}$ recommended surgical release before 1 year of age to prevent and reverse any cranial deformity because this deformity appears on the cranium and cranial base during early life. We performed surgical release at 6 months of age for CMT children who did not show a significant reduction of thickness of the SCM, and who still had moderate limitation of the cervical rotation range and craniofacial asymmetry after 6 weeks of stretching exercise.

Botulinum toxin injection was performed for the patients who still had a considerably shortened SCM 
despite of receiving stretching exercise. Injected botulinum toxin causes the SCM to paralyze, making the SCM easier to be stretched, and it lessens the size of the muscle mass.

Oleszek et al. ${ }^{17}$ reported that they used botulinum toxin type A in the treatment of children with CMT and who failed to improve their range of motion with stretching exercise. A total of 27 children who were 10.1 months of age (range: 6-18 months of age) received botulinum toxin type A injections into only their SCM (29.7 units), their trapezius muscle (28.3 units), or both (55 units). Seventy-four percent of the children had improved cervical rotation or head tilt after the injections and $7 \%$ of the children experienced transient adverse events (mild dysphagia and neck weakness).

Collins and Jankovic ${ }^{16}$ reported that they injected 150 units of botulinum toxin type A into the SCM of children who were 8 months of age, but the effect was negligible, and they also reported that only 2 out of 6 adults with CMT and who were injected with botulinum toxin type A showed improvement. Because of the reported negative results of botulinum toxin injection for adult patients with CMT, research on the safety of injecting botulinum toxin into infants is needed.

In this study, if neck motion limitation was not observed after stretching exercise, then the treatment was discontinued. Clinically, we observed that as a child grew, the mass size lessened, so we considered that the stretching exercise did not directly have an effect on lessening the mass size.

The ultrasonographic-assessed disappearance of the mass was reported to be delayed by an average of 2 weeks as compared to the clinical disappearance of the mass, ${ }^{30}$ and it is known that the normal muscle volume was increased at the periphery, and muscle fibers were regenerated within the mass with the decrease in fibrosis of CMT. ${ }^{30,31}$

This study was a retrospective study with several limitations. We had a small number of patients and we did not include other potential prognostic factors of CMT, such as ultrasonographic information like the echogenicity. Furthermore, the linear regression analysis of the duration of stretching exercises for CMT with the difference of thickness between the SCMs showed a $\mathrm{R}^{2}$ of 0.166 , indicating the relatively low power of the model. Therefore, further studies on other potential prognostic factors of CMT are required in addition to the thickness

\section{of SCM.}

In spite of these limitations, we believe that this report shows the direct relationship between the thickness of the SCM and the prognosis of CMT, and we suggest that if the thickness of the mass-side SCM is larger by $8.8 \mathrm{~mm}$ as compared to the normal side, or the shortened SCM thickness is $>14.01 \mathrm{~mm}$, the prognosis for the stretching exercise is poor. In addition, in the previous studies with an older age group, the researchers generally used the ratio of the different SCMs rather than depth difference as an indicator of CMT. In this study, the age was restricted to $<3$ months, which is a narrow age range. Therefore, the difference of thickness of the SCMs as well as the ratio of the different SCMs could be used as an effective indicator for the prognosis of this age group.

\section{CONCLUSION}

Children with a thicker SCM of the tilted side seem to require a longer duration of stretching exercise and other therapeutic interventions, in addition to stretching exercise for CMT, which indicates that the thickness of the SCM seems to be one of the prognostic factors of CMT.

\section{REFERENCES}

1. Yim SY, Lee IY, Park MC, Kim JH. Differential diagnosis and management of abnormal posture of the head and neck. J Korean Med Assoc 2009; 52: 705-718

2. Chen MM, Chang HC, Hsieh CF, Yen MF, Chen TH. Predictive model for congenital muscular torticollis: analysis of 1021 infants with sonography. Arch Phys Med Rehabil 2005; 86: 2199-2203

3. Cheng JC, Au AW. Infantile torticollis: a review of 624 cases. J Pediatr Orthop 1994; 14: 802-808

4. Cheng JC, Tang SP, Chen TM, Wong MW, Wong EM. The clinical presentation and outcome of treatment of congenital muscular torticollis in infants-a study of 1,086 cases. J Pediatr Surg 2000; 35: 1091-1096

5. Do TT. Congenital muscular torticollis: current concepts and review of treatment. Curr Opin Pediatr 2006; 18: 26-29

6. Ling CM, Low YS. Sternomastoid tumor and muscular torticollis. Clin Orthop Relat Res 1972; 86: 144-150

7. Stevens P, Downey C, Boyd V, Cole P, Stal S, Edmond J, Hollier L. Deformational plagiocephaly associated 
with ocular torticollis: a clinical study and literature review. J Craniofac Surg 2007; 18: 399-405

8. Suzuki S, Yamamuro T, Fujita A. The aetiological relationship between congenital torticollis and obstetrical paralysis. Int Orthop 1984; 8: 175-181

9. Cho KH, Kim JY, Lee IY, Yim SY. Congenital muscular torticollis in siblings: a case report and literature review. J Korean Acad Rehab Med 2009; 33: 731-734

10. Sonmez K, Turkyilmaz Z, Demirogullari B, Ozen IO, Karabulut R, Bagbanci B, Basaklar AC, Kale N. Congenital muscular torticollis in children. ORL J Otorhinolaryngol Relat Spec 2005; 67: 344-347

11. Davids JR, Wenger DR, Mubarak SJ. Congenital muscular torticollis: sequela of intrauterine or perinatal compartment syndrome. J Pediatr Orthop 1993; 13: 141-147

12. Thompson F, McManus S, Colville J. Familial congenital muscular torticollis: case report and review of the literature. Clin Orthop Relat Res 1986; 202: 193-196

13. Cheng JC, Tang SP, Chen TM. Sternocleidomastoid pseudotumor and congenital muscular torticollis in infants: a prospective study of 510 cases. J Pediatr 1999; 134: 712-716

14. Brans J, Aramideh M, Bosch A, Speelman H. Late presentation of congenital muscular torticollis: a nondystonic cause of torticollis. J Neurol 1996; 243: 354-356 15. Singer C, Green BA, Bruce JH, Bowen BC, Weiner WJ. Late presentation of congenital muscular torticollis: use of MR imaging and CT scan in diagnosis. Mov Disord 1994; 9: 100-103

16. Collins A, Jankovic J. Botulinum toxin injection for congenital muscular torticollis presenting in children and adults. Neurology 2006; 67: 1083-1085

17. Oleszek JL, Chang N, Apkon SD, Wilson PE. Botulinum toxin type a in the treatment of children with congenital muscular torticollis. Am J Phys Med Rehabil 2005; 84: 813-816

18. Joyce MB, de Chalain TM. Treatment of recalcitrant idiopathic muscular torticollis in infants with botulinum toxin type a. J Craniofac Surg 2005; 16: 321-327

19. Lim D, Kwon W, Cha SW, Yoo H, Lim S, Park JM, Kim MS. The sonographic correlation between the sternocleidomastoid muscle thickness and the prognosis of congenital muscular torticollis. J Korean Soc Radiol 2009; 60: 133-138
20. Cheng JC, Metreweli C, Chen TM, Tang S. Correlation of ultrasonographic imaging of congenital muscular torticollis with clinical assessment in infants. Ultrasound Med Biol 2000; 26: 1237-1241

21. Tang SF, Hsu KH, Wong AM, Hsu CC, Chang CH. Longitudinal followup study of ultrasonography in congenital muscular torticollis. Clin Orthop Relat Res 2002; 403: 179-185

22. Cheng JC, Wong MW, Tang SP, Chen TM, Shum SL, Wong EM. Clinical determinants of the outcome of manual stretching in the treatment of congenital muscular torticollis in infants. A prospective study of eight hundred and twenty-one cases. J Bone Joint Surg Am 2001; 83-A: 679-687

23. Hsu TC, Wang CL, Wong MK, Hsu KH, Tang FT, Chen HT. Correlation of clinical and ultrasonographic features in congenital muscular torticollis. Arch Phys Med Rehabil 1999; 80: 637-641

24. Tatli B, Aydinli N, Caliskan M, Ozmen M, Bilir F, Acar G. Congenital muscular torticollis: evaluation and classification. Pediatr Neurol 2006; 34: 41-44

25. Celayir AC. Congenital muscular torticollis: early and intensive treatment is critical. A prospective study. Pediatr Int 2000; 42: 504-507

26. Wolfort FG, Kanter MA, Miller LB. Torticollis. Plast Reconstr Surg 1989; 84: 682-692

27. Wei JL, Schwartz KM, Weaver AL, Orvidas LJ. Pseudotumor of infancy and congenital muscular torticollis: 170 cases. Laryngoscope 2001; 111: 688-695

28. Cheng JC, Tang SP. Outcome of surgical treatment of congenital muscular torticollis. Clin Orthop Relat Res 1999; 362: 190-200

29. Yu CC, Wong FH, Lo LJ, Chen YR. Craniofacial deformity in patients with uncorrected congenital muscular torticollis: an assessment from threedimensional computed tomography imaging. Plast Reconstr Surg 2004; 113: 24-33

30. Dudkiewicz I, Ganel A, Blankstein A. Congenital muscular torticollis in infants: ultrasound-assisted diagnosis and evaluation. J Pediatr Orthop 2005; 25: 812-814

31. Lin JN, Chou ML. Ultrasonographic study of the sternocleidomastoid muscle in the management of congenital muscular torticollis. J Pediatr Surg 1997; 32: 1648-1651 